Bond University

Research Repository

\title{
Effectiveness of a School-Based Emotional Freedom Techniques Intervention for Promoting Student Wellbeing
}

Stapleton, Peta; Mackay, Eleanor; Chatwin, Hannah; Murphy, Daniel; Porter, Brett; Thibault, Sally; Sheldon, Terri; Pidgeon, Aileen

Published in:

Adolescent Psychiatry

DOI:

10.2174/2210676607666171101165425

Licence:

Unspecified

Link to output in Bond University research repository.

Recommended citation(APA):

Stapleton, P., Mackay, E., Chatwin, H., Murphy, D., Porter, B., Thibault, S., Sheldon, T., \& Pidgeon, A. (2017).

Effectiveness of a School-Based Emotional Freedom Techniques Intervention for Promoting Student Wellbeing. Adolescent Psychiatry, 7(2), 112-126. https://doi.org/10.2174/2210676607666171101165425

\section{General rights}

Copyright and moral rights for the publications made accessible in the public portal are retained by the authors and/or other copyright owners and it is a condition of accessing publications that users recognise and abide by the legal requirements associated with these rights.

For more information, or if you believe that this document breaches copyright, please contact the Bond University research repository coordinator. 
Effectiveness of a School-Based Emotional Freedom Techniques Intervention for Promoting

Student Wellbeing

Peta Stapleton*1, Eleanor Mackay ${ }^{1}$, Hannah Chatwin ${ }^{1}$, Daniel Murphy ${ }^{1}$, Brett Porter $^{2}$, Sally Thibault $^{1}$, Terri Sheldon ${ }^{2}$, and Aileen Pidgeon ${ }^{1}$

${ }^{1}$ Bond University, Queensland, Australia, ${ }^{2}$ The Lakeside Rooms, Gold Coast, Queensland, 4229, Australia

*Address correspondence to this author at School of Psychology, Bond University, Gold Coast, Queensland, 4229, Australia.

Telephone: +61 755952 515, Facsimile: +61 755952 540, pstaplet@bond.edu.au

Abstract

In academic settings, fear of failure and associated emotional difficulties are common and often result in maladaptive behaviours, which often lead to failure or lowered scholastic achievement. Higher levels of self-esteem and resilience have been shown to protect against fear of failure and emotional difficulties, and predict improved academic outcomes in students. However, few studies have investigated the efficacy of group intervention methods aimed at improving self-esteem and resilience. This non-randomised universal intervention represents the first Australian study of the efficacy of a group Emotional Freedom Techniques (EFT) treatment program within high schools, aimed at increasing student self-esteem and resilience, and decreasing fear of failure and emotional difficulties. The EFT intervention groups $(N=204)$ were drawn from two different school cohorts. Results showed a significant improvement in fear of failure, whereby fears were significantly lower from pre-intervention to 12-month follow-up. Findings also indicated a significant main effect of time for emotional and behavioural difficulties, however post hoc tests indicated no statistically significant changes between the time points measured. No significant changes were observed in measures of self-esteem or resilience. The results suggested that EFT might be an effective group intervention for some students decreasing their fear of failure; however, further research is required. 
Keywords: Emotional Freedom Techniques; students; wellbeing; self-esteem; resilience 
Effectiveness of a School-Based Emotional Freedom Techniques Intervention for Promoting Student Wellbeing

Anxiety and fear of failure in academic settings often lead to significantly reduced performance in the short-term, and poorer life outcomes in the long-term for students who otherwise have the personal characteristics necessary for success, such as intelligence and creativity (Martin, 2010). Chronic underachievement at school is associated with truancy and drop out, dysfunctional behaviour, disengagement from school, and interpersonal difficulties with teachers (Martin, 2010). Long-term underachievement after graduation or school dropout can result in decreased physical and mental health, lesser professional achievement and income, unemployment, and dysfunctional interpersonal relationships. Conversely, higher levels of self-esteem and resilience protect students from negative responses to scholastic demands and generally predict superior academic outcomes (Martin, 2010; Waxman, Gray, \& Padron, 2003).

Academic stress affects a large proportion of students; however, providing effective interventions to such a large population presents logistical problems. This study represented the first Australian research into the effectiveness of an emerging therapeutic technique known as Emotional Freedom Techniques (EFT) in improving high school student outcomes on measures of fear of failure, strengths and difficulties, self-esteem, and resilience.

The potential benefits of EFT for high school students were investigated for a number of reasons. The technique has reduced potential for emotional harm compared to traditional interventions (Flint, Lammers, \& Mitnick, 2006), making it amenable to delivery in large groups without the need for prior screening procedures. Delivery to large groups is more cost effective than individual therapy, and also has the benefit of potentially helping students who are not identified as needing psychological intervention, yet still experience significant academic stress. A number of other factors also indicate that EFT is brief and easy to learn, 
can be utilised by participants in their own time, and has the potential for immediate individual benefits (Church, Pina, Reategui, \& Brooks, 2012).

Treatment in the current universal trial aimed to improve four participant characteristics: global self-esteem, resilience (ability to adapt to change and cope with stress), total difficulties and fear of failure (cognitive, motivational, and relational appraisals of failure). All four characteristics were utilised as outcome variables in the present study. Research has shown each to play a role in influencing academic success.

\section{Self Esteem}

The definition of self-esteem has been subject to continuing discussion throughout the relevant literature and researchers have highlighted the need to distinguish between global and specific self-esteem (Rosenberg, Schooler, Schoenbach, \& Rosenberg, 1995). Here, selfesteem refers to a favourable or unfavourable attitude towards oneself (Rosenberg, 1965). Hence, the present study operationalised self-esteem as a global characteristic measured by the Rosenberg Self-esteem Scale (RSES; Rosenberg, 1965). Although it is commonly believed high self-esteem enhances academic performance, there is overall inconsistency in the literature exploring this relationship. Rosenberg, Schooler, and Schoenbach (1989) reported that, although school grades appeared to affect self-esteem, there was no significant effect of global self-esteem in enhancing academic performance. These results are consistent with several longitudinal studies, finding no significant role of general self-esteem in the promotion of school performance or academic grades (Bachman \& O’Malley, 1977; Ciarrochi, Heaven, \& Davies, 2007; Helmke \& van Aken, 1995; Muijs, 1997). Hence, previous studies showing a modest positive correlation between self-esteem and school performance, are typically interpreted as indicating that high self-esteem is, at least partly, the result of good school performance (Baumeister, Campbell, Krueger, \& Vohs, 2003). According to Rosenberg et al. (1989), the implication of this finding is that efforts to develop 
self-esteem in order to promote academic performance are pointless. However, there does appear to be a significant contribution of self-esteem to other important areas of adolescent development.

Past research demonstrates a positive and significant relationship between global selfesteem and broad measures of psychological wellbeing (Rosenberg et al., 1995). In fact, Campbell (1981) found self-esteem was one the strongest predictors of wellbeing. More recently, Baumeister and colleagues (2003) concluded happiness was one of the strongest and most advantageous correlates of high self-esteem in their review of the literature. Cheng and Furnham (2003) corroborated these findings, demonstrating self-esteem, as measured by the RSES (Rosenberg, 1965), predicted nearly $50 \%$ of the total variance on a self-report measure of happiness. Although the literature has not firmly established causation, Baumeister and colleagues (2003) concluded the evidence indicates self-esteem leads to high levels of happiness. Paradise and Kernis (2002) also examined the degree to which self-esteem predicted scores on Ryff's (1989) scales of psychological wellbeing, finding self-esteem positively predicted higher scores on three of six subscales: autonomy, environmental mastery, and purpose in life. Hence, it seems protective for an individual to establish and maintain a sense of their worth.

Longitudinal research conducted by Di Giunta et al. (2013) demonstrated that selfesteem was a significant predictor of self-efficacy, which in turn predicted academic success. The study utilised a staggered, multiple cohort design, and participants were selected from two junior high schools. The two cohorts analysed were assessed at age 14, 16, and 19. Results demonstrated that conscientiousness, openness, and self-esteem at age 16 were all interrelated and significantly predicted self-efficacy levels, which in turn exerted a significant influence on senior high school grades. This research demonstrates that although higher academic achievement may reciprocally influence self-esteem levels, self-esteem plays a 
significant role in determining academic success even when prior achievements are taken into account.

\section{Fear of Failure}

Research has shown fear of failure to be intrinsically linked to self-esteem. Fear of failure refers to appraisal of the potential impact of failure on individual and public perceptions of competence, and emotional responses to these appraisals. Research by Martin, Marsh and Debus (2003) demonstrated that fear of failure often results in two maladaptive coping strategies, including self-handicapping and defensive pessimism. The authors investigated these constructs in a one-year longitudinal study utilising a sample of 328 Australian undergraduate university students. Results indicated that higher levels of selfesteem significantly predicted higher academic grades, and that higher levels of selfhandicapping and defensive pessimism predicted lower academic performance. These findings demonstrated support for the role of self-esteem in predicting academic success, but did not provide direct measurement of fear of failure.

Research directly linking fear of failure to self-handicapping and other adverse academic outcomes was reported by De Castella, Byrne and Covington (2013). The authors investigated the effects of fear of failure in two student samples: 1,423 Japanese and 643 Australian high school students. Results demonstrated that higher levels of fear of failure significantly predicted lower grades and increased levels of self-handicapping, truancy, and disengagement. The results of these studies indicate that both self-esteem and fear of failure are appropriate outcome variables and targets for intervention in the current universal intervention.

\section{Resilience}

Resilience has also been shown to be an important factor in predicting student engagement in maladaptive strategies to protect self-worth. A study by Martin (2013) 
demonstrated the importance of resilience for scholastic engagement in a sample of 918 Australian high school students ranging from 11 to 19 years of age. Two types of resilience were investigated: academic buoyancy (capacity to overcome everyday academic challenges and setbacks) and academic resilience (capacity to overcome acute or chronic educational adversity). Findings revealed that higher levels of academic buoyancy significantly predicted lower levels of anxiety, uncertain control, and failure avoidance. Higher levels of academic resilience significantly predicted lower levels of self-handicapping and disengagement. Martin and Marsh (2006) investigated the same construct of academic resilience as the aforementioned study in a sample of 402 Australian high school students. Path analyses demonstrated that academic resilience significantly predicted three outcomes: enjoyment of school, class participation, and self-esteem. The results demonstrated that resilience is an important factor in scholastic engagement, which is a requisite for academic success.

\section{Strengths and Difficulties}

The fourth outcome measure in the present study measured student social and emotional difficulties, due to the strong relationship between social and emotional health and academic success. Strong evidence of this link was provided by Durlak, Weissberg, Dymnicki, Taylor, and Schellinger (2011), who conducted a meta-analysis of the effects of 213 school-based controlled trials of social and emotional learning (SEL) programs on academic performance. The results demonstrated that SEL participants exhibited significantly better academic performance (11-percentiles higher) than control group members, and had significantly better social and emotional skills, behaviours, and attitudes. These results indicate not only a link between social and emotional health and academic success, but that these characteristics are not static and can be improved through intervention.

\section{EFT Description and Mechanisms of Action}


EFT is one of a group of therapies within the domain of energy psychology. The technique is based on the principles of acupuncture, which posit that negative emotions in the body are due to a disturbance in the body's energy meridian fields (Feinstein, 2008). EFT incorporates elements of exposure therapy, cognitive behaviour therapy, and somatic stimulation to target negative thoughts and feelings. The technique involves tapping on meridian (acupressure) points on the face and body while focusing on a specific negative thought (the source of the emotion) through self-affirmation and acceptance statements (Church, 2013).

The effect of EFT on stress biochemistry was empirically tested in a randomised controlled trial conducted by Church, Yount and Brooks (2012). The authors randomly assigned 83 non-clinical participants to one of an EFT, psychotherapy (supportive interview), or no intervention group. The results showed that the EFT group experienced a significant $24.39 \%$ decrease in cortisol levels when compared to the psychotherapy and no intervention groups, indicating that the application of EFT resulted in clinically significant reduced stress levels. Additionally, the EFT group exhibited significant improvements in anxiety and depression scores. These results indicated that EFT has a direct impact on stress biochemistry and may be of particular benefit for emotional disorders involving over-arousal of the limbic systems, such as anxiety and stress.

Reductions in stress and anxiety after utilising EFT could be solely due to the exposure component of the technique, which is common to a variety of therapeutic approaches. Fox (2013) conducted a randomised controlled study comparing normal EFT to a control condition where EFT protocols were followed except for the use of tapping and vocalised self-acceptance statements. The results showed that the EFT group exhibited significantly higher academic enjoyment and hope, and significantly lower anger and shame, than the control group at post-intervention. These findings indicate that the acupoint tapping 
and vocalised self-acceptance statements are an active component of EFT intervention rather than inert placebo, and distinguish the technique from other exposure therapies. Overall, the body of research indicates that EFT functions as an effective exposure technique with direct effects on physical stress.

\section{EFT Effectiveness Research}

Although EFT is a relatively recent treatment approach, a comprehensive review of the literature conducted by Feinstein (2008) suggested that EFT has reached the lower threshold of the required American Psychology Association criteria to be identified as an evidence-based therapy for phobias and weight loss. A number of randomised controlled studies have demonstrated the effectiveness of EFT in treating a variety of physical and psychological conditions. These include PTSD (Church et al., 2013), fear of public speaking (Jones, Thornton, \& Andrews, 2011), depression (Church, de Asis, \& Brooks, 2012), pain in fibromyalgia patients (Brattberg, 2008), food craving strength (Stapleton, Sheldon, Porter, \& Whitty, 2011), and phobias (Salas, Brooks, \& Rowe, 2011).

A limited number of studies have been conducted investigating EFT treatment of both anxiety and depression in high school and university students. Research by Benor, Ledger, Toussaint, Hett and Zaccaro (2009) investigated the effectiveness of EFT in treating a sample of 15 Canadian university students suffering from moderate or severe test anxiety. The authors employed a double-blind controlled trial, where five participants were allocated to each of an EFT, Cognitive Behavioural Therapy, or Wholistic Hybrid technique (derived from Eye Movement Desensitisation and Reprocessing) treatment group. All treatment groups showed similar improvements on measures of test anxiety, demonstrating that EFT resulted in similar improvements in anxiety as the other empirically validated interventions.

Furthermore, the authors reported that the EFT group obtained the same improvements in two 
sessions as the CBT group obtained in five sessions. Despite the small sample size, these results provided support for the potential cost effective implementation of EFT interventions in a school setting, due to the speed of improvement when compared to a CBT intervention.

EFT has also been compared to Progressive Muscular Relaxation (PMR) intervention in an academic setting. Sezgin, Ozcan, and Church (2009) compared the effectiveness of EFT and PMR for reducing test anxiety in a sample of 312 high school students with higher test anxiety, as measured by the Test Anxiety Inventory (TAI; Speilberger, 2010). Both groups received a single treatment session, and were instructed to use the techniques at home. The results showed that both groups experienced reduced test anxiety, and that the EFT group exhibited significantly lower test anxiety (on both the worry and emotionality subscales) when compared to the PMR group. No significant difference between groups on the sample examination scores was observed. Scores increased for both groups; however, this could be due to practice effects. This research demonstrated the effectiveness of EFT for reducing test anxiety in high school-aged students similar in age to those sampled in the present study; however, the study only included those identified as having high levels of anxiety prior to intervention, and does not allow for interpretations of the possible effectiveness of EFT for those with sub-clinical test anxiety.

Jain and Rubino (2012) also examined the effectiveness of EFT for treating test anxiety in a randomised controlled trial involving 168 American undergraduate students suffering from self-reported test anxiety. The participants were allocated to one of EFT, Diaphragmatic Breathing (DB), or a no-intervention control group. The EFT and DB groups received two group sessions of two hours each. The results showed that both intervention groups showed significant improvements in anxiety on all measures when compared to the control group, and that all gains were maintained at follow-up. This study provides support for the use of EFT to treat test anxiety in students, but like the previously mentioned study 
only recruited participants with high levels of test anxiety. This precludes generalisation to general student populations with less than severe test anxiety.

Other research has demonstrated the effectiveness of EFT for treating depression in student samples. Church, de Asis, and Brooks (2012) conducted a randomised controlled trial to investigate EFT for treating depression in a sample of university students. In this study, 30 participants with moderate to severe depression were randomly assigned to treatment (EFT) and control (no-intervention) groups. The EFT group received four sessions of group EFT training. Post-intervention measurements using the BDI showed that the EFT group demonstrated significant improvement compared to the control group. While these results indicate support for the use of EFT in treating depression in an academic setting, it should be noted that the sample consisted of those with high levels of depression, and the results cannot be generalised to a general student sample, as used in the present study.

Finally, Boath, Stewart, and Carryer (2013) conducted a pilot study to test the effectiveness of EFT in reducing anxiety and increasing performance in a group of 52 university students. The results showed significant reductions in distress and anxiety, but not depression, for the students who used EFT in their own time. This research indicated the potential effectiveness of the use of EFT to target academic-related stress and anxiety, and that the technique could be beneficial after only one training session. The lack of improvement in depression is consistent with the aforementioned theory that EFT primarily targets the arousal components of emotional distress.

\section{Overview of Current Study}

The aim of the current study was to investigate the effectiveness of an EFT intervention in improving levels of self-esteem, resilience, strengths and difficulties, and fear of failure in a group of Year 10 High School students. Previous research has demonstrated the effectiveness of EFT in treating students identified with severe test anxiety and clinical 
depression; however, this study examined the usefulness of EFT in a general student population, whose academic performance was also impacted by stress related to academic demands. Additionally, this study expanded upon previous research by focussing treatment and measurement not only on student anxiety (fear of failure), but including three other domains which have been shown in previous research to impact on academic success: selfesteem, resilience, and strengths and difficulties. This multidimensional approach recognised that the aetiology of academic fear and failure is multifaceted in nature (Martin, 2010) and that a broader treatment approach may yield better results, particularly because the intervention was delivered in a group modality. Short- and long-term retention effects were also explored over several time points, including 3- and 12-month follow-up.

It was expected that scores on the measures of self-esteem and resilience would significantly increase at post-intervention, indicating increased self-esteem and resilience. It was also predicted that scores on the measures of fear of failure, and social and emotional difficulties, would significantly decrease at post-intervention, indicating decreased difficulties and fear of failure. Finally, it was expected that post-intervention improvements for all four measures would be significantly maintained at 3- and 12-month follow up.

\section{Method}

\section{Participants}

Participants consisted of 204 Year 10 students from two Gold Coast high schools; College $1(n=80)$ and the College 2 who were a control group $(n=124)$. Of the included participants from College 1, 58\% were females and $42 \%$ were males ranging in age from 14 to 16 years old $(M=14.74, S D=0.54)$. Of the included participants from College $2,66 \%$ were females and $34 \%$ were males ranging in age from 13 to 16 years old $(M=14.89, S D=$ $0.73)$.

\section{Materials}


Rosenberg Self-Esteem Scale (RSES; Rosenberg, 1965). The RSE is a 10-item selfreport measure of global and unidimensional self-esteem for individuals of high school age and up and was considered appropriate for this study as it is regularly used in treatment outcome studies . It was also deemed the length of the scale was suitable for adolescents, and the reliability of the scale has been assessed amongst an Australian teenage sample of 352 boys and 411 girls, where Rigby and Cox (1996) found adequate alpha values of .73 for boys and .74 for girls. Items consist of five positive and five negative statements regarding feelings about the self, and respondents answer on a 4-point Likert-type scale ranging from "strongly agree" to "strongly disagree". Total scores range from 10 to 40 after summing all item scores, with higher scores representing higher levels of self-esteem. A sample item includes "I certainly feel useless at times". The factor structure, internal consistency $(\alpha=.89)$, and cultural validity of the RSE have been evidenced in previous studies (i.e., Rosenberg, 1965; Schmitt \& Allik, 2005). Evidence of construct and criterion validity of the RSE in a large high school student sample was obtained by Bagley, Bolitho, and Bertrand (2007). The Cronbach's alphas for the RSES ranged between .89 and .94 across all time points in the present study.

Conners-Davidson Resilience Scale (CD-RISC; Connor \& Davidson, 2003). The CD-RISC is a 25-item self-report measure of state resilience for individuals aged 12 and older, and was chosen as it distinguishes between those with greater and lesser resilience. The scale demonstrates that resilience is modifiable and can improve with treatment, with greater improvement corresponding to higher levels of global improvement. Each CD-RISC item represents a statement regarding perceived resilience and adaptability, and participants respond using a 5-point Likert-type scale ranging from 0 (not true at all) to 4 (true nearly all of the time). Responses are based on experiences over the past month. A sample item includes "I am able to adapt when changes occur". Windle, Bennet, and Noyes (2011) reported that 
the CD-RISC consistently demonstrated good to excellent internal consistency, and acceptable convergent validity with other resilience scales. Cronbach's alphas ranged between .89 and .94 across all time points, for the CD-RISC in the present study.

Strengths and Difficulties Questionnaire for ages 11-17 (SDQ; Goodman, 1997). The SDQ is a brief 25-item self-report measure of emotional and behavioural difficulties for children and adolescents and is unique in that it also screens a person's strengths. Participants respond to both positive and negative statements about their emotional and behavioural experiences over the previous month, on a 3-point Likert-type scale ranging from 0 (not true) to 2 (certainly true). A sample item includes "I think before I do things". This measure includes five subscales: emotional problems, conduct problems, hyperactivity, peer problems, and pro-social behaviours. The current study utilised the total difficulties score, which is calculated by summing all subscale scores except the pro-social scale. Achenbach et al. (2008) found that the total difficulties scale demonstrates good internal consistency $(\alpha=.80)$ and test-retest reliability $(r=.79)$ in British and Australian samples. Moreover, the criterion validity of the scale has been validated in previous studies (e.g., Ziaian, Anstiss, Antouiou, Baghurst, \& Sawyer, 2012). Cronbach's alphas for the SDQ total difficulties score ranged between .75 and .88 across all time points in the present study.

\section{Performance Failure Appraisal Index-Short Form (PFAI; Conroy, Willow, \&}

Metzler, 2002). The PFAI is a 5-item Likert-type measure of cognitive, motivational, and relational appraisals associated with fear of failure. It was designed to measure fear as a multidimensional construct, and rather than an attempt to fit fear of failure into a situational condition, the authors considered it was a function derived from the interaction of individuals in their environment. The present study deemed this appropriate for the experiences of students. Participants respond to five statements regarding beliefs about failure on a scale ranging from -2 (do not believe at all) to 2 (believe $100 \%$ of the time). Total scores range 
from -2 to 2, and higher scores represent greater fear of failure and this general fear of failure can be interpreted as the strength of an individual's belief that failure is generally associated with aversive consequences. A sample item includes "When I am failing, I worry about what others will think of $m e^{\prime \prime}$. Previous studies have revealed that the PFAI possesses adequate reliability and construct validity (e.g., Conroy, Coatsworth, \& Kaye, 2007). The Cronbach's alphas for the instrument ranged between .74 and .85 across all time points in the present study.

Weekly program evaluation. After each session participants were asked to fill in an anonymous evaluation form to provide feedback on their experience to the facilitators. The form consisted of a comments section and three questions answered on a 7-point Likert-type scale, including questions surrounding how useful the EFT information and skills were, how easy to understand the EFT information was, and how confident participants felt in using the EFT information and skills covered.

\section{Procedure}

Ethical approval to conduct this study was granted by the University Human Research Ethics Committee. The State Government Department of Education, Training, and Employment also granted ethical approval. Gatekeeper approval was obtained from each school principal. At each site the Year 10 students were presented with information about the program in a group, and supplied with an explanatory statement and parental consent form. Parents were supplied with a parental information form.

At College 1, 80 students participated in the EFT intervention. College $2(n=79)$ acted as a control group and completed the pre-questionnaire package, then waited the length of treatment before completing the EFT intervention. All intervention data was collapsed into one group at the study conclusion for analysis. Figure 1 describes the research design and flow of participants during the experiment. 
Participants completed the package of measures immediately prior to treatment groups commencing (pre-intervention), after the completion of the final treatment session (postintervention), and 3-months after treatment finished. College 2 also participated in 12-month follow-up. All participants used a unique identifying code (first four letters of mother's maiden name and child's year of birth) on all measures for confidentiality and so that pre-, post-, and follow-up could be matched for data analysis. Additionally, after each EFT session participants were asked to evaluate the program to provide social validity data and feedback for the facilitators.

Overview of Treatment Program. The EFT intervention for academic fear and failure was delivered over five weekly sessions of 75 minutes each, during normal school hours. The program facilitators were a registered clinical psychologist and a psychotherapist and both were qualified EFT practitioners. The school guidance officer was also present at each session. Treatment protocols and fidelity plans for the EFT intervention were formed prior to commencing and each practitioner signed these each session for adherence to the manual.

Session 1 consisted of an overview of EFT and how to use it, including group skills practice. Students were encouraged to engage in a personal experiment to test the effectiveness of EFT and establish their own truth about the technique. Session 2 commenced with discussion related to using EFT to target academic fears and anxiety. EFT was then used to target five common negative self-statements (e.g., "School is a waste of time"). In Session 3, two interactive activities were introduced to keep the students engaged in tapping: a ball game and Tangrams (dissection puzzle) activity. Three common barriers to effectively using the technique were targeted using EFT (e.g., "Doubting that EFT can or will work").

Session 4 focussed on using EFT to target limiting beliefs and its application to areas such as academic and sporting performance (such as confidence and performance anxiety). 
Four common negative self-statements relating to success were targeted (e.g., "Success will make me stand out"). The final session involved using EFT to assist with three areas: (1) student's limiting expectations of themselves both at school and in other areas of life; (2) perceptions of other people's expectations regarding participant behaviour and achievements; and (3) goal setting for the future, including doubts about achieving these goals.

\section{Design}

This study represented a non-randomised universal intervention, utilising both within and between-subject designs. There were two independent variables: time (pre-intervention, post-intervention, 3-month follow-up, and 12-month follow-up) and school (College 1 vs. College 2). Dependent variables were self-esteem (Rosenberg Self-esteem Scale), resilience (Conner-Davidson Resilience Scale), difficulties (total difficulties score on the Strengths and Difficulties Questionnaire), and fear of failure (Performance Failure Appraisal InventoryShort Form). As the two intervention groups were not randomised from the same sample, independent samples $t$-tests were used to compare the groups on each outcome measure at baseline to ensure the groups were not significantly different.

\section{Results}

\section{Data Diagnostics}

A power analysis using the G*Power application developed by Faul, Erdfelder, Lang, and Buchner (2007) demonstrated that the current study had sufficient participants to detect any significant treatment effects. Between groups comparison of post-treatment effects was conducted using a 2 x 2 mixed design repeated measures analysis of variance (rANOVA) for each dependent variable. Follow-up effects were investigated utilising one-way rANOVA and post hoc comparisons with Bonferroni adjustments. All results were interpreted using a decision criterion of $\alpha=.05$.

\section{Preliminary Analyses}


Table 1 shows the inter-correlations for all dependent variables at each point in time. Significant correlations were observed amongst self-esteem, resilience, difficulties, and fear of failure, as expected in an adolescent sample of high school students (Martin \& Marsh, 2003). Table 2 shows the descriptive statistics for each dependent variable in the EFT groups and each point in time. Mean scores on the RSE and SDQ were in the normal clinical range at each point in time. Mean scores on the PFAI at each point in time were within one standard deviation of the normative mean published in the user manual (Conroy, 2002), and comparable with descriptive statistics of student populations (Conroy et al., 2002). Mean scores on the CD-RISC in this study were similar to those found in populations suffering from generalised anxiety (Conner \& Davidson, 2003), demonstrating that this student cohort may have been experiencing significant academic or other stress.

Independent samples $t$-tests comparing the EFT and collapsed groups on each dependent variable at pre-intervention were performed, in order to determine whether the groups shared similar characteristics before treatment commenced. Table 3 demonstrates that there were no significant differences in outcome variables present at pre-treatment. These results indicate that the EFT groups can be meaningfully collapsed to assess follow-up treatment effects.

\section{Treatment Effects}

To explore changes within the EFT treatment groups over time, outcome variable scores at each time point (pre, post, 3-months, and 12-months) were explored using rANOVA for each dependent variable and subsequent post hoc paired comparisons, with Bonferroni adjustments for multiple comparisons. Analyses comparing follow-up scores were based on a smaller sample due to attrition before 3- and 12-month measurements and missing data. Table 4 shows the mean and standard deviation for each dependent variable at each point in time. 
Mauchly's test of sphericity was significant $(p<.01)$ for self-esteem, resilience, and difficulties. As suggested by Tabachnick and Fidell (2007), the Huynh-Feldt Epsilon adjustment was utilised to determine the significance of relevant $F$-tests. A significant main effect of time was found for difficulties, $F(12.61,25.99)=3.04, p=.40, \eta^{2}=.09$, power $=$ .65 , and fear of failure, $F(3.00,17.32)=3.37, p=.02, \eta^{2}=.09$, power $=.75$. Significant main effects of time were not obtained for resilience, $F(2.68,31.23)=0.57, p=.62, \eta^{2}=.02$, power $=.16$, or self-esteem, $F(3.00,5.22)=0.52, p=.67, \eta^{2}=.02$, power $=.15$.

Table 5 shows the results of the post hoc paired comparisons performed for the EFT group. Despite the aforementioned main effect of time for difficulties, pairwise comparisons revealed no statistically significant differences between any of the time points. For the main effect of time for fear of failure, pairwise comparisons revealed that the only statistically significant change was from pre-test to 12-month follow-up $(p=.020)$.

\section{Discussion}

This study aimed to investigate whether a brief treatment program would be beneficial to a general student population. The research represented the first Australian trial of EFT in a school setting and was conducted within normal school hours. This represented an important strength of the trial, as it did not impose on student's personal time which may have decreased the time available to complete schoolwork and increased academic stress during the intervention.

Findings of the current study indicated fear of failure significantly reduced from preintervention to 12-month follow-up, which may imply that treatment gains are not restricted to the active phase of treatment and may positively impact on student emotional health and academic performance into the future. This finding also suggests that EFT may have a delayed intervention effect for certain groups of symptoms, as recent research have similarly established (Church \& Brooks, 2014; Stapleton et al., 2012). 
The range of possible scores on the PFAI is limited, which may have resulted in more pronounced clinical changes from pre- to post-intervention or 3-month follow-up points being undetectable statistically. Another reason for the lack of significant differences at early measurement points could be that the PFAI measures cognitive appraisal of failure, rather than unhelpful cognitions and behaviours that this construct commonly drives, such as selfhandicapping and defensive pessimism. It is possible that students who undertook the EFT program still experienced fear of failure, but was able to utilise EFT to accept this fear and engage in more adaptive behaviours in response. An improvement in such behaviours would not be adequately captured by the outcome measures used in this study, but if present would lead to increased academic performance despite similar levels of fear of failure.

Although the results also demonstrated an overall main effect of time for emotional and behavioural difficulties, post hoc tests revealed no statistically significant difference between any of the time points measured. Inspection of the means indicated that the range of scores (12.27 to 13.7 across all time points) revealed that these were in the normal range, therefore detecting change after an intervention is more difficult. It is possible that these gains may have been understated due to the broad nature of the construct measured by the SDQ, whereby measurement may have missed more subtle changes in difficulties specific to academic endeavour. Another potential reason for the lack of significant improvement observed between time points is the wide range of problems in these areas that teenagers commonly experience (Deas \& Brown, 2006). Not only was there limited time to address individualised issues in addition to academic fear and anxiety concerns, the public nature of the intervention would likely prevent most students from suggesting a particular problem to tap on within this domain. Even if a common problem was successfully targeted, the SDQ measures a wide range of problems within the one instrument. 
Findings of the current study also indicated that the EFT groups exhibited no significant improvements in self-esteem or resilience at post-intervention or follow-up points, which is largely inconsistent with previous research demonstrating the effectiveness of EFT in promoting improvements in psychological variables among student populations (e.g., Benor, Ledger, Toussaint, Hett, \& Zaccaro, 2009; Boath, Stewart, \& Carryer, 2013). This may imply that EFT interventions for large groups of students may not be viable in terms of overall effectiveness and cost effectiveness; however, it should be noted that student baseline resilience scores indicated the presence of anxiety levels commonly found in populations suffering from generalised anxiety disorder (Conner \& Davidson, 2003). Considering this information, any considerable increase in resilience levels in this highly anxious adolescent population indicates that EFT may result in positive outcomes for at least some students in this age group. Inspection of the means indicated that the self-esteem scores were in the normal range and thus may explain why no differences occurred during the intervention.

A possible reason for the lack of improvement observed for self-esteem and resilience was the large group modality employed for the treatment program. While common negative beliefs regarding academic performance were identified and treated, more individualised intervention was limited; targeted negative thoughts and emotions may not have been specific to some students. Secondly, the participants were of an age when many are self-conscious and fear appearing foolish in front of their peers. The tapping and vocalised components of the program may have caused some anxiety and resulted in a lack of motivation to engage fully in the session and invest in the process emotionally.

\section{Limitations}

Overall the decrease in fear of failure and overall effect of time for difficulties indicate that EFT may be of benefit to at least some students. But there were limitations in the current study. The two groups were of very similar age and gender composition, and did not differ 
significantly on the measurement of outcome variables at baseline; however, it is possible that there were differing academic demands and schedules between study sites, and other such confounding variables that dilute study findings (e.g. demographic variables). The study did seek demographic information from all parents, however only $40 \%$ of all parents returned their surveys. It is possible this information may also have had an effect on the study outcomes. It is recommended a number of changes could be included in future studies investigating the effectiveness of EFT interventions for academic success. Researchers could include academic performance measures, such as student grades or specific academic testing before and after program delivery. Including these assessments would not only allow direct examination of the effect on EFT on scholastic performance, but with a large enough sample would allow path analyses of the interactions between all mediating variables, such as selfconcept and academic resilience. Results of such studies would then allow for more precise applications of EFT that target the beliefs and associated behaviours that most significantly influence academic performance. The current study did not receive permission from the education department to access these but this would be invaluable in the future.

Overall, this study does provide valuable information about the application and effectiveness of the technique delivered in a group modality. It indicated that EFT has the potential to decrease fear of failure and emotional/behavioural difficulties in some students. In future, more specific outcome measures may demonstrate positive changes in additional domains. The potential improvements in student functioning, ease of teaching the method, and group delivery mode may highlight that further research into the effectiveness of group EFT interventions is warranted, as it may offer students significant benefits with low risks and time demands.

\section{ABOUT THE AUTHORS}


Peta Stapleton, $\mathbf{P h D}$ is an Associate Professor and the Program Director of the Masters of Clinical Psychology at Bond University, Australia.

Eleanor Mackay is a Clinical Psychologist and works for Autism Spectrum Australia (Aspect).

Hannah Chatwin is a Clinical Psychologist and works at Monash ELMHS (Early in Life Mental Health Service).

Daniel Murphy is a Clinical Psychologist and works in private practice.

Brett Porter is n EFT practitioner, counsellor, and group therapy specialist and works at The Lakeside Rooms, Australia.

Sally Thibault is an EFT Practitioner and works in private practice.

Terri Sheldon is a Clinical, Counselling, Educational and Developmental Psychologist., and Adjunct Senior Teaching Fellow in the School of Psychology, Bond Unievrsity, Australia.

Aileen Pidgeon, PhD is an Assistant Professor and $4^{\text {th }}$ year coordinator in the School of Psychology, Bond University, Australia.

\section{CONFLICT OF INTEREST}

None of the authors have any conflict of interest relevant to this manuscript.

\section{ACKNOWLEDGMENTS}

This study was funded under a faculty grant with Bond University. We wish to also acknowledge the schools who participated in the study. 


\section{References}

Achenbach, T. M., Becker, A., Döpfner, M., Heiervang, E., Roessner, V., Steinhausen, H. C., \& Rothenberger, A. (2008). Multicultural assessment of child and adolescent psychopathology with ASEBA and SDQ instruments: research findings, applications, and future directions. Journal of Child Psychology and Psychiatry, 49, 251-275. doi:10.1111/j.1469-7610.2007.01867.x

Bagley, C., Bolitho, F., \& Bertrand, L. (2007). Norms and construct validity of the Rosenberg Self-Esteem Scale in Canadian high school populations: Implications for counseling. Canadian Journal of Counseling and Psychotherapy, 31. Retrieved from http://www.cjc-rcc.ucalgary.ca/cjc/index.php/rcc/article/view/85

Benor, D. J., Ledger, K., Toussaint, L., Hett, G., \& Zaccaro, D. (2009). Pilot study of EFT, WHEE and CBT for treatment of test anxiety in university students. Explore: The Journal of Science and Healing, 5, 338-340. doi:10.1016/j.explore.2009.08.001

Benetti C., \& Kambouropoulos, N. (2006). Affect-regulated indirect effects of trait anxiety and trait resilience on self-esteem. Personality and Individual Differences, 41, 341352.

Boath, E., Stewart, A, \& Carryer, A. (2013). Tapping for success: A pilot study to explore if Emotional Freedom Techniques (EFT) can reduce anxiety and enhance academic performance in university students. Innovative Practice in Higher Education, 1. Retrieved from http://journals.staffs.ac.uk/index.php/ipihe/article/view/33

Brattberg, G. (2008). Self-administered EFT (Emotional Freedom Techniques) in individuals with fibromyalgia: a randomized trial. Integrative Medicine, 7, 30-35. Retrieved from http://www.varkstaden.se/pdf_filer/EFT_article.pdf 
Church, D. (2013). Clinical EFT as an evidence-based practice for the treatment of psychological and physiological conditions. Psychology, 4, 645-654. Retrieved from http://file.scirp.org/Html/35751.html

Church, D., De Asis, M. A., \& Brooks, A. J. (2012). Brief group intervention using emotional freedom techniques for depression in college students: A randomized controlled trial. Depression Research and Treatment, 2012. doi:10.1155/2012/257172

Church, D., Hawk, C., Brooks, A. J., Toukolehto, O., Wren, M., Dinter, I., \& Stein, P. (2013). Psychological trauma symptom improvement in veterans using emotional freedom techniques: A randomized controlled trial. The Journal of nervous and mental disease, 201, 153-160. doi:10.1097/NMD.0b013e31827f6351

Church, D., Pina, O., Reategui, C., \& Brooks, A. (2012). Single-session reduction of the intensity of traumatic memories in abused adolescents after EFT: A randomized controlled pilot study. Traumatology: An International Journal, 18, 73-79. Retrieved from http://emofree.ch/files/single_session_abused_adolescents.pdf

Church, D., Yount, G., \& Brooks, A.J. (2012). The effect of emotional freedom techniques on stress biochemistry: A randomized controlled trial. The Journal of Nervous and Mental Disease, 200, 891-896. doi:10.1097/NMD.0b013e31826b9fc1

Connor, K. M., \& Davidson, J. R. (2003). Development of a new resilience scale: The Connor-Davidson Resilience Scale (CD-RISC). Depression and Anxiety, 18, 76-82. Retrieved from http://static1.squarespace.com/

Conroy, D. E., Coatsworth, J. D., \& Kaye, M. P. (2007). Consistency of fear of failure score meanings among 8-to 18-year-old female athletes. Educational and Psychological Measurement, 67, 300-310. doi:10.1177/0013164406288174 
Conroy, D. E., Willow, J. P., \& Metzler, J. N. (2002). Multidimensional fear of failure measurement: The Performance Failure Appraisal Inventory. Journal of Applied Sport Psychology, 14, 76-90. doi:10.1080/10413200252907752

Deas, D., \& Brown, E. S. (2006). Adolescent substance abuse and psychiatric comorbidities. Journal of Clinical Psychiatry, 67, 18-23. Retrieved from http://www.yodaa.org.au/sites/default/files/Adolescent\%20DD\%202006.pdf

De Castella, K., Byrne, D., \& Covington, M. (2013). Unmotivated or motivated to fail? A cross-cultural study of achievement motivation, fear of failure, and student disengagement. Journal of Educational Psychology, 105, 861-880. doi:10.1037/a0032464

Di Giunta, L., Alessandri, G., Gerbino, M., Kanacri, P. L., Zuffiano, A., \& Caprara, G. V. (2013). The determinants of scholastic achievement: The contribution of personality traits, self-esteem, and academic self-efficacy. Learning and Individual Differences, 27, 102-108. doi:10.1016/j.lindif.2013.07.006

Durlak, J. A., Weissberg, R. P., Dymnicki, A. B., Taylor, R. D., \& Schellinger, K. B. (2011). The impact of enhancing students' social and emotional learning: A meta-analysis of school based universal interventions. Child development, 82, 405-432. doi:10.1111/j.1467-8624.2010.01564.x

Faul, F., Erdfelder, E., Lang, A., \& Buchner, A. (2007). G*Power 3: A flexible statistical power analysis program for the social, behavioral, and biomedical sciences. Behavior Research Methods, 39, 175-191. Retrieved from http://www.gpower.hhu.de

Feinstein, D. (2008). Energy psychology: A review of the preliminary evidence. Psychotherapy: Theory, Research, Practice, Training, 45, 199-213. Retrieved from https://www.innersource.net/ep/epresearch.html 
Flint, G. A., Lammers, W., \& Mitnick, D. G. (2006). Emotional Freedom Techniques: A safe treatment intervention for many trauma based issues. Journal of aggression, maltreatment \& trauma, 12, 125-150. doi:10.1300/J146v12n01_07

Fox, L. (2013). Is acupoint tapping an active ingredient or an inert placebo in Emotional Freedom Techniques (EFT)? A randomized controlled dismantling study. Energy Psychology, 5, 15-28. Retrieved from http://energypsychologyjournal.org/wpcontent/uploads/2014/02/Accupoint-tapping_Placebo_in_EFT_Study_EPJ5.2_byFox.pdf

Goodman, R. (1997). The Strengths and Difficulties Questionnaire: A research note. Journal of Child Psychology and Psychiatry, 38, 581-586. doi:10.1111/j.14697610.1997.tb01545.x

Jain, S., \& Rubino, A. (2012). The effectiveness of Emotional Freedom Techniques (EFT) for optimal test performance: A randomized controlled trial. Energy Psychology: Theory, Research, \& Treatment, 4, 13-24. doi:10.9769.EPJ.2012.4.2.SJ

Jones, S., Thornton, J., \& Andrews, H. (2011). Efficacy of Emotional Freedom Techniques (EFT) in reducing public speaking anxiety: A randomized controlled trial. Energy Psychology: Theory, Research, \& Treatment, 3, 19-32. Retrieved from http://espace.library.curtin.edu.au/R?func=dbin-jump-full\&local_base=gen01era02\&object_id=173102

Jørgensen I.E., \& Seedat S. (2008). Factor structure of the Connor-Davidson Resilience Scale in South African adolescents. Int J Adolesc Med Health, 20, 23-32.

Martin, A. (2010). Building classroom success: eliminating academic fear and failure. New York, NY: Continuum International Publishing Group 
Martin, A. (2013). Academic buoyancy and academic resilience: Exploring 'everyday'and 'classic'resilience in the face of academic adversity. School Psychology International, 34, 488-500. doi:10.1177/0143034312472759

Martin, A. J., \& Marsh, H. W. (2006). Academic resilience and its psychological and educational correlates: A construct validity approach. Psychology in the Schools, 43, 267-281. doi:10.1002/pits.20149

Martin, A. J., Marsh, H. W., \& Debus, R. L. (2003). Self-handicapping and defensive pessimism: A model of self-protection from a longitudinal perspective. Contemporary Educational Psychology, 28, 1-36. doi:10.1016/S0361-476X(02)00008-5

Rosenberg, M. (1965). Society and the adolescent self-image. Princeton, NJ: Princeton University Press

Salas, M. M., Brooks, A. J., \& Rowe, J. E. (2011). The immediate effect of a brief energy psychology intervention (Emotional Freedom Techniques) on specific phobias: A pilot study. Explore: The Journal of Science and Healing, 7, 155-161. doi:10.1016/j.explore.2011.02.005

Schmitt, D. P., \& Allik, J. (2005). Simultaneous administration of the Rosenberg Self-Esteem Scale in 53 nations: exploring the universal and culture-specific features of global selfesteem. Journal of personality and social psychology, 89, 623-642. doi:10.1037/00223514.89.4.623

Sezgin, N., Ozcan, B., Church, D., (2009) The effect of two psychophysiological techniques (progressive muscular relaxation and emotional freedom techniques) on test anxiety in high school students: A randomized blind controlled study. International Journal of Healing and Caring, 9. Retrieved from http://www.eftuniverse.com/ 
Stapleton, P. B., Sheldon, T., Porter, B., \& Whitty, J. (2011). A randomised clinical trial of a meridian-based intervention for food cravings with six-month follow-up. Behaviour Change, 28, 1-16. Retrieved from http://epublications.bond.edu.au/hss_pubs/631/ Tabachnick \& Fidell (2007). Experimental design using ANOVA. Belmont, CA: Duxbury Tabachnick \& Fidell (2013). Using multivariate statistics (6th Ed.). Boston: Pearson

Waxman, H. C., Gray, J. P., and Padron, Y. N. (2003). Review of research on educational resilience: Research report. Washington, DC: Institute of Education Sciences.

Windle, G., Bennett, K. M., \& Noyes, J. (2011). A methodological review of resilience measurement scales. Health and quality of life outcomes, 9, 1-18. Retrieved from http://www.biomedcentral.com/content/pdf/1477-7525-9-

8.pdf?www.mrc.ac.uk/Ourresearch/ResearchInitiatives/LLHW/index.htm

Yu X., Lau J.T.F., Mak W.W.S., Zhang J., Lui W.W.S., \& Zhang, J. (2011). Factor structure and psychometric properties of the Connor-Davidson Resilience Scale among Chinese adolescents. Comprehensive Psychiatry, 52, 218-224.

Ziaian, T., de Anstiss, H., Antoniou, G., Baghurst, P., \& Sawyer, M. (2012). Resilience and its association with depression, emotional and behavioural problems, and mental health service utilisation among refugee adolescents living in South Australia. International Journal of Population Research, 2012. doi:10.1155/2012/485956 


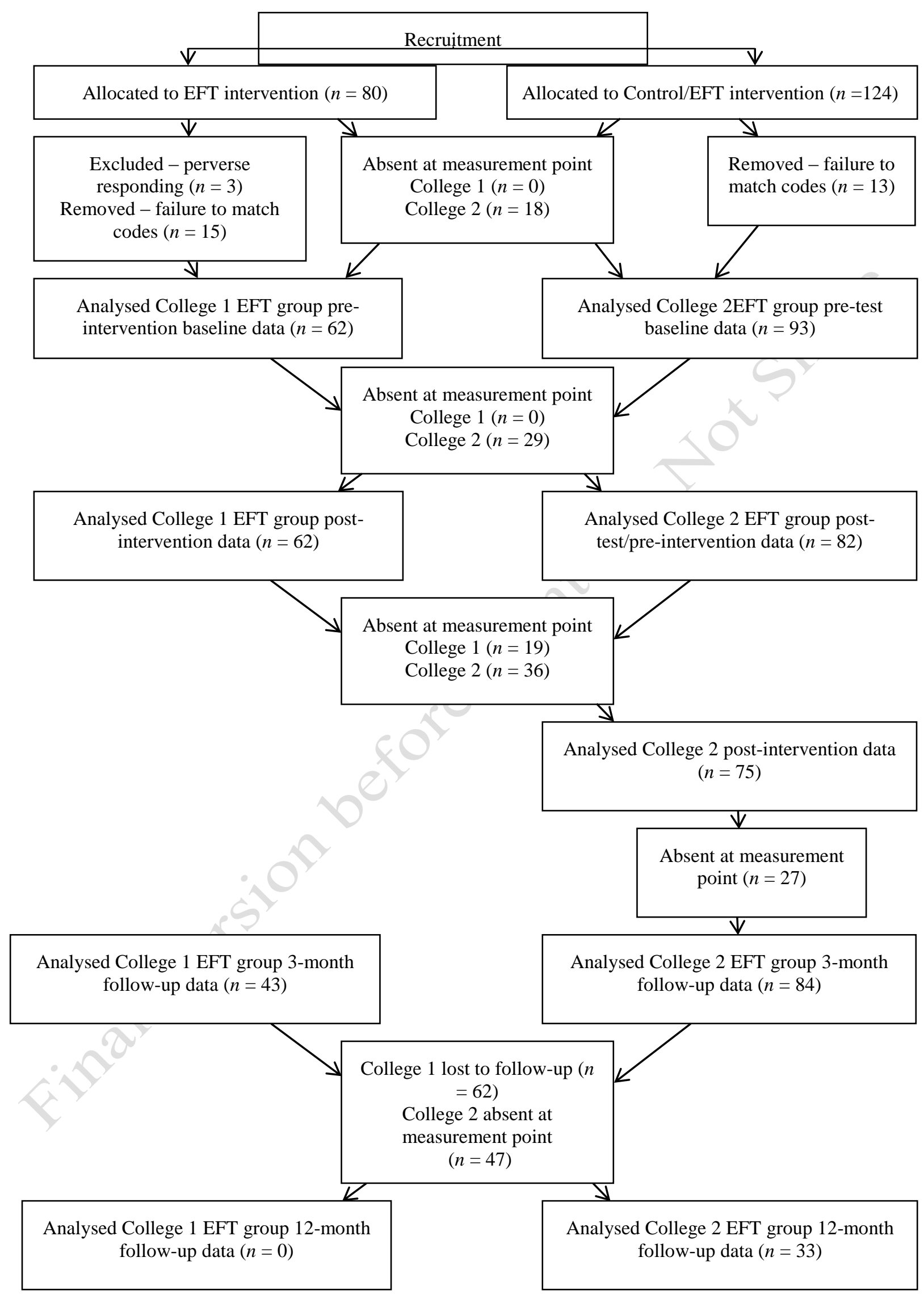

Figure 1. CONSORT statement depicting the research design and flow of participants through the controlled clinical trial. 
Table 1

Summary of Intercorrelations between RSES, CD-RISC, SDQ, and PFAI-S scores at Pre-test, Post-test/Pre-intervention, Post-intervention, 3-month Follow-up, and 12-month Follow-up

\begin{tabular}{|c|c|c|c|}
\hline Variable & 2 & 3 & 4 \\
\hline \multicolumn{4}{|l|}{ 1. RSES } \\
\hline Pre-test & $.588 * *$ & $-.453 * *$ & $-.398 * *$ \\
\hline Post-test/Pre-intervention & $.709 * *$ & $-.565 * *$ & $-.440 * *$ \\
\hline Post-intervention & $.644 * *$ & $-.541 * *$ & $-.466 * *$ \\
\hline 3-month follow-up & $.761 * *$ & $-.556 * *$ & $-.643 * *$ \\
\hline 12-month follow-up & $.837 * *$ & $-.671 * *$ & $-.644 * *$ \\
\hline \multicolumn{4}{|l|}{ 2. CD-RISC } \\
\hline Pre-test & - & $-.425 * *$ & $-.264^{*}$ \\
\hline Post-test/Pre-intervention & - & $-.454 * *$ & $-.337 * *$ \\
\hline Post- intervention & - & $-.405^{* *}$ & $-.307 *$ \\
\hline 3-month follow-up & - & $-.50 * *$ & $-.54 * *$ \\
\hline 12-month follow-up & & $-.471 * *$ & $-.602 * *$ \\
\hline \multicolumn{4}{|l|}{ 3.SDQ } \\
\hline Pre-test & & - & $-.388 * *$ \\
\hline Post-test/Pre-intervention & & - & $.273 * *$ \\
\hline Post- intervention & & - & $.387 * *$ \\
\hline 3-month follow-up & & - & $.504 * *$ \\
\hline 12-month follow-up & & - & $.498 * *$ \\
\hline \multicolumn{4}{|l|}{ 4. PFAI-S } \\
\hline Pre-test & & & - \\
\hline Post-test/Pre-intervention & & & - \\
\hline Post-intervention & & & - \\
\hline 3-month follow-up & & & - \\
\hline 12-month follow-up & & & - \\
\hline
\end{tabular}

Note. RSES = Rosenberg Self-esteem Scale; CD-RISC = Conner-Davidson Resilience Scale; SDQ $=$ Strengths and Difficulties Questionnaire; PFAI-S = Performance Failure Appraisal Inventory - Short Form.

$* p<.05, * * p<.01$ 
Table 2

Means and Standard Deviations of RSES, CD-RISC, SDQ, and PFAI-S Scores for EFT Groups Across All Time Points

\begin{tabular}{|c|c|c|c|c|c|c|c|}
\hline \multicolumn{4}{|c|}{ EFT Group } & \multicolumn{4}{|c|}{ Control Group } \\
\hline & Pre- & Post- & 3-month & Pre- & Post- & 3-month & 12-month \\
\hline & intervention & intervention & follow-up & intervention & intervention & follow-up & follow-up \\
\hline Variable & $M(S D)$ & $M(S D)$ & $M(S D)$ & $M(S D)$ & $M(S D)$ & $M(S D)$ & $M(S D)$ \\
\hline \multirow[t]{2}{*}{ RSES } & 28.15 & 30.08 & 30.42 & 29.66 & 29.80 & 28.85 & 28.81 \\
\hline & $(6.00)$ & $(6.29)$ & $(5.93)$ & $(6.44)$ & $(6.50)$ & $(6.91)$ & $(6.78)$ \\
\hline \multirow[t]{2}{*}{ CD-RISC } & 65.10 & 67.53 & 68.37 & 64.65 & 65.46 & 65.89 & 66.79 \\
\hline & (13.28) & (13.35) & (13.17) & (15.63) & $(14.68)$ & $(16.40)$ & $(14.83)$ \\
\hline \multirow[t]{2}{*}{ SDQ } & 13.25 & 13.70 & 12.28 & 12.27 & 12.74 & 13.60 & 13.26 \\
\hline & $(5.63)$ & $(5.15)$ & $(5.13)$ & $(5.85)$ & (6.07) & $(7.38)$ & $(6.58)$ \\
\hline \multirow[t]{2}{*}{ PFAI-S } & -0.22 & -0.08 & -0.49 & -0.17 & -0.22 & -0.01 & 0.20 \\
\hline & $(0.98)$ & $(1.03)$ & (1.12) & $(1.19)$ & $(0.99)$ & $(1.05)$ & $(0.91)$ \\
\hline
\end{tabular}

Note. RSES = Rosenberg Self-esteem Scale; CD-RISC = Conner-Davidson Resilience Scale; SDQ = Strengths and Difficulties Questionnaire; PFAI-S = Performance Failure Appraisal Inventory - Short Form. 
Table 3

Dependent Variable T-test Results for EFT Groups at Pre-intervention

\section{$95 \% \mathrm{CI}$}

\begin{tabular}{lcccccc} 
Variable & $t$ & $d f$ & $p$ & $S E$ & Lower & Upper \\
\hline RSES & 1.434 & 140 & .154 & 1.058 & -0.574 & 3.609 \\
CD-RISC & -0.180 & 140 & .857 & 2.479 & -5.349 & 4.455 \\
SDQ & -0.998 & 136 & .320 & 0.988 & -2.947 & 0.970 \\
PFAI-S & -0.038 & 131 & .970 & 0.169 & -0.341 & 0.328
\end{tabular}

Note. RSES = Rosenberg Self-esteem Scale; CD-RISC = Conner-Davidson Resilience Scale; SDQ $=$ Strengths and Difficulties Questionnaire; PFAI-S = Performance Failure Appraisal Inventory - Short Form; SE = Standard Error Difference; CI = Confidence Interval. 
Table 4

Means and Standard Deviations for each Dependent Variable at Pre, Post, and Follow-up within the EFT Group Participants with Follow-up Data

\begin{tabular}{lcccc}
\hline $\begin{array}{l}\text { Dependent } \\
\text { variable }\end{array}$ & $\begin{array}{c}\text { Pre-intervention } \\
\text { Self-esteem }\end{array}$ & $\begin{array}{c}\text { Post-intervention } \\
M(S D)\end{array}$ & $\begin{array}{c}\text { 3-month } \\
\text { follow-up } \\
M(S D)\end{array}$ & $\begin{array}{c}\text { 12-month } \\
\text { follow-up } \\
M(S D)\end{array}$ \\
\hline $\begin{array}{l}\text { Resilience } \\
\text { Difficulties }\end{array}$ & $62.15(18.05)$ & $63.91(16.97)$ & $63.30(16.39)$ & $64.24(14.88)$ \\
Fear of failure & $12.12(6.66)$ & $12.42(6.61)$ & $12.27(7.66)$ & $13.91(7.13)$ \\
\hline Note. $N=33$ & $-0.15(0.89)$ & $0.07(0.87)$ & $0.30(1.07)$ & $0.36(0.83)$ \\
\hline
\end{tabular}

Note. $N=33$ 
Table 5

Post Hoc Paired Comparisons, with Bonferroni Adjustments, Comparing each Dependent Variable at each Point in Time (Pre, Post, 3-months,

and 12-months)

\begin{tabular}{|c|c|c|c|c|c|c|c|c|c|c|c|c|}
\hline & \multicolumn{2}{|c|}{ Pre vs. Post } & \multicolumn{2}{|c|}{ Post vs. $3 \mathrm{~m}$} & \multicolumn{2}{|c|}{ Pre vs. $3 \mathrm{~m}$} & \multicolumn{2}{|c|}{ Post vs. $12 \mathrm{~m}$} & \multicolumn{2}{|c|}{ Pre vs. $12 \mathrm{~m}$} & \multicolumn{2}{|c|}{$3 \mathrm{~m}$ vs. $12 \mathrm{~m}$} \\
\hline & $\mathrm{Md}$ & $p$ & $\mathrm{Md}$ & $p$ & $\mathrm{Md}$ & $p$ & $\mathrm{Md}$ & $p$ & $\mathrm{Md}$ & $P$ & $\mathrm{Md}$ & $p$ \\
\hline RSES & -0.15 & 1.00 & 0.36 & 1.00 & 0.21 & 1.00 & 0.91 & 1.00 & 0.76 & 1.00 & 0.55 & 1.00 \\
\hline CD-RISC & -1.76 & 1.00 & 0.61 & 1.00 & -1.15 & 1.00 & -0.33 & 1.00 & -2.09 & 1.00 & -0.94 & 1.00 \\
\hline SDQ & -0.30 & 1.00 & 0.15 & 1.00 & -0.15 & 1.00 & -1.49 & .227 & -1.79 & .129 & -1.64 & .207 \\
\hline PFAI & -0.22 & .650 & -0.22 & .929 & -0.44 & .079 & -0.29 & .315 & -0.50 & $.021 *$ & 0.44 & .079 \\
\hline
\end{tabular}

$* p<.05$

Note $. \mathrm{Md}=$ Mean difference. RSES = Rosenberg Self-esteem Scale; CD-RISC = Conner-Davidson Resilience Scale; SDQ = Strengths and

Difficulties Questionnaire; PFAI-S = Performance Failure Appraisal Inventory - Short Form 
